\title{
Lebensqualität in Deutschland und Europa
}

Die wirtschaftliche Entwicklung in Deutschland ist über die letzten Jahre günstiger als in anderen EU-Mitgliedstaaten verlaufen und die Einkommen liegen am oberen Ende der Verteilung. Allerdings hängt die Lebensqualität in einem Land von weiteren Faktoren über das Gesamteinkommen hinaus ab. Geht die positive Wirtschaftsentwicklung beispielsweise mit Verschlechterungen bei Vereinbarkeitsfragen oder gar in der lokalen Umwelt einher? Wie steht es um Spaltungen und soziale Spannungen zwischen Bevölkerungsgruppen? Solchen Fragen gehen wir auf Basis neuer Umfragedaten zur Lebensqualität in Europa nach. ${ }^{1}$

HANS DUBOIS, ESZTER SANDOR, ERIKA MEZGER

\section{Einleitung}

Der vorliegende Report soll einen Eindruck von der Lebensqualität in Deutschland vermitteln. Er basiert auf der Europäischen Erhebung zur Lebensqualität (EQLS), die von Ende 2011 bis Anfang 2012 in allen 27 EU-Mitgliedsstaaten und sieben anderen Ländern durchgeführt wurde (Eurofound 2012a). Der Befragung gingen zwei frühere Erhebungsrunden in den Jahren 2003 und 2007 voraus. Im vorliegenden Bericht geht es nicht nur um die Vorstellung der jüngsten Ergebnisse, sondern auch um eine Analyse der beobachteten Veränderungen, vor allem im Vergleich zu 2007. ${ }^{2}$ Die Untersuchung der allgemeinen Lebensqualität in Deutschland und der Vergleich mit dem übrigen Europa konzentrieren sich auf folgende Grundaspekte der Lebensqualität: 1) Lebensstandard und materielle Entbehrung, 2) subjektives Wohlbefinden und psychische Gesundheit, 3) Beschäftigung, Privatleben und Vereinbarkeit von Berufs- und Privatleben, 4) Wohneigentum, Wohnbedingungen und Wohnumfeld, 5) öffentliche Dienste, Gesundheitswesen und Gesundheitszustand sowie 6) Zustand der Gesellschaft.

Die EQLS ist eine repräsentative Befragung von Personen im Alter ab 18 Jahren, die seit sechs Monaten oder länger ihren Wohnsitz im jeweiligen Land haben. In Deutschland wurden 3.000 Personen befragt, mehr als in allen anderen EU-Mitgliedstaaten. Die Befragung erfolgte in Form eines persönlichen Gesprächs in der Privatwohnung der Befragten. Die überwiegende Mehrheit der Interviews fand im letzten Quartal 2011 statt.

Für Deutschland war der Wirtschaftskontext von 2007 bis 2011 vergleichsweise günstig. Während nahezu alle Mitgliedstaaten in mindestens zwei Jahren dieses Zeitraums einen wirtschaftlichen Abschwung erlebten, hatte Deutschland lediglich 2009 eine reale Minderung des Bruttoinlandsprodukts zu verzeichnen (Eurostat 2013); in den übrigen Jahren legte die deutsche Wirtschaft bei realer Betrachtung zu. Die durchschnittliche jährliche Wachstumsrate betrug für Deutschland in den genannten fünf Jahren 1,3\%, für Gesamteuropa hingegen nur 0,6 \%. Diese Entwicklung hat die Sonderstellung Deutschlands unter den wohlhabenderen EU-Mitgliedstaaten weiter gefestigt. Allerdings hängt Lebensqualität von mehr als nur dem durchschnittlichen Einkommen in einem Land ab. Der Beitrag untersucht weitere Einflussfaktoren und legt dabei besonderen Wert auf subjektive Erfahrungsaspekte, die auf die jeweilige Lebensqualität wirken.

Übersetzung aus dem Englischen von Ana Elisa Eskuche.

2 Eurofound wird Mitte 2013 eine vertiefende Analyse zu „Tendenzen bei der Lebensqualität in Europa 2003-2012" veröffentlichen. 


\section{Lebensstandard und materielle Entbehrung}

\subsection{Deckung des Haushaltsbedarfs}

Trotz des relativen wirtschaftlichen Wohlstands kann sich ein signifikanter Anteil der Bevölkerung Deutschlands nur mit Mühe finanziell über Wasser halten: 30 \% der Einwohner Deutschlands sagen, dass ihr Haushalt nur unter „einigen“ bis "großen“ Schwierigkeiten mit seinen Einkünften auskomme. Trotz der insgesamt günstigen Wirtschaftsentwicklung hat sich der Anteil der Befragten, die angeben, nicht oder nur knapp über die Runden zu kommen, im Zeitraum 2007-2011 von 26 auf 30 \% erhöht.

Allgemein berichten ältere Personen seltener als jüngere von Schwierigkeiten bei der Deckung des Haushaltsbedarfs. Während in den Altersgruppen von 18 bis 24 und von 25 bis 34 Jahren $35 \%$ der Befragten angeben, nur mit Mühe zurechtzukommen, liegt dieser Anteil bei den 35- bis 49-Jährigen bei $30 \%$, bei den 50 - bis 64-Jährigen bei $28 \%$ und für Personen ab 65 Jahren bei $17 \%$. Dennoch wäre es ein Trugschluss zu glauben, dass ältere Leute keine finanziellen Sorgen haben. Eine kürzliche Untersuchung von Eurofound (2012b) stellte zum Beispiel fest, dass ein beträchtlicher Anteil der Rentner in Deutschland gezwungen ist, seine Rente durch bezahlte Arbeit aufzubessern.

\subsection{Materielle Entbehrung}

Der Begriff materielle Entbehrung bezeichnet die Unfähigkeit, Ausgaben für Dinge zu bestreiten, die zum Grundbedarf gerechnet werden. Zur Beurteilung, ob und in welchem Umfang materielle Entbehrung vorliegt, wurde gefragt, ob der jeweilige Haushalt sich sechs grundlegende Dinge leisten kann. Auf diese Weise lässt sich finanzielle Not genauer beurteilen als anhand des Haushaltseinkommens: Es ist erfassbar, was den Befragten unabhängig von ihrem Einkommen und ihren Vermögensverhältnissen derzeit nicht möglich ist. Konkret wurde gefragt, ob die Befragten beziehungsweise der Haushalt sich folgende sechs Dinge leisten konnten:

1. ihr Haus bzw. ihre Wohnung angemessen warmzuhalten bzw. zu heizen

2. einmal im Jahr einen einwöchigen Urlaub weg von $\mathrm{zu}$ Hause (nicht bei Verwandten)

3. jeden zweiten Tag eine Mahlzeit mit Fleisch, Huhn oder Fisch

4. abgenutzte Möbel zu ersetzen

5. neuwertige Kleidung (anstelle gebrauchter Kleidungsstücke) zu kaufen

6. mindestens einmal im Monat die Familie oder Freunde auf ein Getränk oder zum Essen einzuladen.

Der Großteil (68\%) der Befragten in Deutschland gab an, sich alle sechs Dinge leisten zu können; nur $1 \%$ kann sich nach eigener Aussage nichts davon leisten. Damit steht Deutschland im europäischen Vergleich eindeutig gut da: $55 \%$ aller Befragten in der EU gaben an, sich alle die aufgeführten Positionen leisten zu können, 3 \% konnten sich keine davon leisten.

Auch wenn man die zeitliche Entwicklung vergleicht, schneidet Deutschland besser ab als die EU insgesamt. 2007 betrug der Anteil der Einwohner, die sich nach eigener Angabe mindestens fünf der sechs Positionen leisten konnten, für Deutschland $79 \%$, im EU-Durchschnitt dagegen nur $74 \%$. Auch 2011 lag der genannte Anteil in Deutschland unverändert bei $79 \%$, während in der EU insgesamt nur noch $67 \%$ sagten, sich mindestens fünf der sechs aufgeführten Positionen leisten zu können. Analog dazu lag im Jahr 2007 der Anteil der Einwohner, die angaben, sich höchstens eins der genannten Dinge leisten zu können, in Deutschland bei $4 \%$, im EU-Durchschnitt bei $5 \% .2011$ hatte sich auch in dieser Hinsicht die Situation in Deutschland nicht verändert (4\%), europaweit dagegen verschlechtert (7\%).

\subsection{Schulden}

Weiterhin erfasst die EQLS für den relevanten Zeitraum Zahlungsrückstände als Indiz für die tatsächliche Unfähigkeit von Haushalten, finanziell über die Runden zu kommen, ohne Zahlungsverpflichtungen zu vernachlässigen. 2007 erfasste die Erhebung zwei typische Zahlungsrückstände. Es wurde gefragt, ob der Haushalt in den letzten zwölf Monaten vor der Befragung zu irgendeinem Zeitpunkt folgende Forderungen nicht termingerecht bezahlen konnte:

1. Miet- oder Hypothekenrate für Wohnung/Haus

2. Rechnungen für Versorgungsleistungen wie Strom, Wasser oder Gas.

Ein Zehntel (10 \%) aller Befragten in Deutschland gab an, mit Mietzahlungen oder Hypothekenraten in Verzug geraten zu sein. Dieser Anteil entspricht dem EU-Durchschnitt. Allerdings hat sich die Situation in Deutschland verbessert (2007: $11 \%$ ), während sie sich in der EU insgesamt verschlechtert hat (2007: $8 \%$ ). Hinsichtlich nicht termingerechter Bezahlung von Versorgungsleistungen unterschied Deutschland sich 2007 nicht wesentlich vom EU-Durchschnitt: 12 bzw. $13 \%$. Im Jahr 2011 sah die Situation in Deutschland unverändert aus (12\%), EU-weit dagegen hatte sie sich verschlechtert (15\%).

2011 wurden zum ersten Mal auch Zahlungsrückstände bei Verbraucherkrediten in die Befragung aufgenommen. Kredite dieser Art dienen typischerweise für den Erwerb von Elektroartikeln, Autos, Möbeln und so weiter. Erfasst wurden auch Kreditlimitüberziehungen, wobei diese potenziell aber auch zur Abdeckung der beiden vorgenannten Arten von Verbindlichkeiten dienen können. In Deutschland berichtet ein höherer Anteil von Haushalten (12\%) als in der EU insgesamt (durchschnittlich $10 \%$ ) von Zahlungsrückständen im Zusammenhang mit Verbraucherkrediten. 
Auf die Frage, an wen sie sich wenden würden, wenn sie dringend $900 €$ benötigten, nennen dennoch nur $7 \%$ aller in Deutschland Befragten einen Dienstleister oder eine Organisation als Anlaufstelle, gegenüber $8 \%$ für die EU. In Deutschland würden sich $84 \%$ an ein Familienmitglied, einen Verwandten, Freund oder Nachbarn oder an eine andere Person wenden. Dieser Prozentsatz übersteigt den EU-Durchschnitt (82\%). Folglich wäre es vermutlich irreführend, wenn die Untersuchung sich auf Zahlungsrückstände gegenüber offiziellen Kreditgebern und Versorgungsunternehmen beschränken würde. Es könnte gut sein, dass informelle Darlehen eine unerwartet große Zusatzrolle spielen.

Die EQLS befasst sich als erste EU-weite Erhebung mit dieser Vermutung. Es wurde gefragt, ob der Haushalt sich mit der Rückzahlung informeller Schulden an Freunde oder an Verwandte außerhalb des Haushalts im Rückstand befunden hatte. In Deutschland gaben $12 \%$ der Befragten an, im Jahr 2011 mit Schuldenrückzahlungen dieser Art in Verzug gewesen zu sein. Dieser Wert liegt deutlich über dem EU-Durchschnitt von $8 \%$. Zudem springt hier der Unterschied zwischen den verschiedenen Einkommensgruppen ins Auge. Von den Angehörigen des untersten Einkommensviertels geben in Deutschland $19 \%$ an, mit Schulden dieser Art im Rückstand gewesen zu sein; im obersten Viertel sind informelle Schuldenrückstände deutlich weniger verbreitet (7\%). Eine mögliche Erklärung hierfür ist, dass Bezieher geringerer Einkommen nur eingeschränkt Zugang zu formellen Krediten oder Darlehen haben.

\section{Subjektives Wohlbefinden, psychische Gesundheit}

Der Begriff „subjektives Wohlbefinden“ beschreibt die Einschätzung der eigenen Lebensqualität durch die Befragten. Als Maßstab dienen Indikatoren aus dem kognitiven Bereich, wie allgemeine Lebenszufriedenheit und Zufriedenheit mit verschiedenen Teilbereichen des Lebens (beispielsweise Gesundheit, Lebensstandard, Familie) sowie Fragen zum individuellen Glücksempfinden und zum Ausmaß positiver und negativer Gefühle und Stimmungslagen.

\subsection{Lebenszufriedenheit und Glücksempfinden}

Auf einer Skala von 1 bis 10 liegt die durchschnittliche Lebenszufriedenheit in Deutschland bei 7,2, das Glücksempfinden bei 7,4 Punkten. Beide Werte entsprechen in etwa dem europäischen Durchschnitt. Üblicherweise stehen beide Indikatoren in einem direkten Zusammenhang zur Wirtschaftsleistung. Das subjektive Wohlempfinden ist aber in Deutschland niedriger als in Ländern mit einem vergleichbaren Einkommensniveau, wie beispielsweise Österreich, die Niederlande oder das Vereinigte Königreich. Anderer- seits ist jedoch hervorzuheben, dass die Werte für Lebenszufriedenheit und Glücksempfinden der Deutschen seit der letzten Erhebung im Wesentlichen stabil geblieben sind, während in vielen anderen europäischen Ländern ein Rückgang zu verzeichnen war.

Im Gegensatz zu einer Vielzahl anderer Länder gibt es in Deutschland hinsichtlich Lebenszufriedenheit und Glücksempfinden keine großen altersbedingten Unterschiede, die Werte für Menschen ab 65 Jahren gleichen den Durchschnittswerten. In der Mehrheit der Mitgliedstaaten, die der EU 2004 oder später beigetreten sind, schneidet diese Bevölkerungsgruppe im Durchschnitt beträchtlich schlechter $\mathrm{ab}$, während in den meisten der Union schon länger angehörenden Ländern die über 65-Jährigen meist deutlich zufriedener und glücklicher sind als der Durchschnitt. Wenn man jedoch zusätzlich nach Einkommensniveau differenziert, zeigen sich auch in Deutschland die älteren Bürger deutlich lebenszufriedener als die Angehörigen jüngerer Bevölkerungsgruppen.

Bei Differenzierung nach verschiedenen Variablen stehen in der EU Gesundheit, Einkommen und Beschäftigungssituation am stärksten mit dem subjektiven Wohlempfinden in Zusammenhang (Eurofound 2012a). Unter Berücksichtigung derselben Variablen stellt man für Deutschland fest, dass der Faktor Arbeitslosigkeit mit der stärksten Abnahme der Lebenszufriedenheit (-1,5 Punkte) korreliert, gefolgt von der Einschätzung der eigenen Gesundheit als schlecht oder sehr schlecht (-1,4 Punkte).

Der Einfluss von Einkommensunterschieden auf das subjektive Wohlbefinden ist höher als im Durchschnitt der EU: Der Unterschied zwischen der Lebenszufriedenheit der Befragten im obersten und im untersten Einkommensviertel ${ }^{3}$ beträgt 1,3 Punkte (EU-Durchschnitt: 1,1), der Unterschied im Glücksempfinden 1,1 Punkte (EU-Durchschnitt: 0,9). Bezieht man weitere Variablen in die Analyse ein, so beläuft sich der Unterschied zwischen dem untersten und dem obersten Viertel auf 0,8 Punkte und entspricht damit in etwa dem Wert, den wir für die gesamte EU beobachten.

\subsection{Optimismus}

Eine weitere Bestimmungsgröße für subjektives Wohlempfinden ist der Optimismus. In Deutschland zeigten sich die Befragten bemerkenswert optimistisch: Der Aussage „Ich sehe der Zukunft optimistisch entgegen“ stimmten $65 \%$ „eher“ oder „,voll und ganz“ zu. EU-weit waren nur $52 \%$ so zuversichtlich. Zudem ist der Anteil der optimistischen Antworten in Deutschland seit der letzten Erhebung gestiegen (2007: 59 \%), während in vielen anderen Ländern ein allgemeiner Rückgang zu verzeichnen war. Möglicher-

3 Der Berechnung der Einkommensviertel wurde das äquivalente Haushaltseinkommen in Euro-Kaufkraftparitäten (KKP) zugrunde gelegt. 
weise lässt sich die vergleichsweise hohe Zuversicht in Deutschland zumindest zum Teil durch die Zufriedenheit mit der Wirtschaftslage erklären, die mit 5,7 Punkten deutlich über dem EU-Durchschnitt von 4,1 liegt und „im Europa nach der Krise“ zu den höchsten Werten zählt.

\subsection{Psychische Gesundheit}

Die EQLS verwendet den WHO-5-Fragebogen der Weltgesundheitsorganisation zur Erfassung des psychischen Wohlbefindens. Der WHO-5-Fragebogen bewertet das psychische Wohlbefinden auf einer Skala von 0 bis 100 anhand der Fragestellung, wie oft in den letzten zwei Wochen nach eigener Einschätzung des Befragten folgende Aussagen zutrafen:

1. Ich war fröhlich und guter Laune

2. Ich habe mich ruhig und entspannt gefühlt

3. Ich habe mich energisch und aktiv gefühlt

4. Ich habe mich beim Aufwachen frisch und ausgeruht gefühlt

5. Mein Alltag war voller Dinge, die mich interessieren.

Das durchschnittliche WHO-5-Ergebnis der Bevölkerung Deutschlands beträgt 65,7 Punkte und liegt damit über dem EU-Durchschnitt von 62,5.

Dieses seelische Wohlbefinden ist stark einkommensabhängig. Das WHO-5-Ergebnis des untersten Einkommensviertels bleibt um 10 Punkte hinter dem des obersten Viertels zurück. Der Unterschied fällt größer aus als in der EU insgesamt. Das WHO-5-Ergebnis von Personen, die ihren Gesundheitszustand als schlecht bezeichnen, liegt um 23 Punkte unter dem derjenigen, die ihre gesundheitliche Verfassung nicht als schlecht beurteilen. Auch dieser Unterschied ist größer als im EU-Durchschnitt. Diese Beobachtungen hinsichtlich des Einflusses von Einkommens- und Gesundheitsunterschieden auf die psychische Gesundheit korrelieren mit den ausgeprägten Unterschieden beim subjektiven Wohlbefinden. Bei der psychischen Gesundheit lässt sich jedoch eine höhere Geschlechtergleichheit feststellen: Während in der EU die Frauen im Schnitt ein schlechteres WHO-5-Ergebnis erzielen als die Männer, ist in Deutschland das durchschnittliche Ergebnis für Frauen und Männer in etwa gleich ( 65 beziehungsweise 66 Punkte).

\section{Beschäftigung, Privatleben und Work-Life-Balance}

Die Einwohner Deutschlands scheinen mit der Vereinbarkeit von Berufs- und Privatleben weniger Probleme zu haben als die übrige EU-Bevölkerung. So sagen zum Beispiel in Deutschland nur $24 \%$ der Befragten, es sei ihnen wegen des Zeitaufwands für den Beruf schwergefallen, ihren fa- miliären Verpflichtungen nachzukommen; der EU-Durchschnitt beträgt $30 \%$. Ein kombinierter Indikator für stressbedingten Konflikt (Eurofound 2012a) verdeutlicht, dass die Einwohner Deutschlands im Vergleich zu denen der meisten anderen Länder sowohl bei der Arbeit als auch zu Hause weniger häufig durch Konflikte zwischen Beruf und Privatleben unter Stress leiden (8 \% im Vergleich zu durchschnittlich $13 \%$ ). Der Anteil der Befragten, die zu Hause, am Arbeitsplatz oder an beiden Orten solchen Stress erleben, ist in Deutschland im Zeitraum 2007- 2011 von 44 auf 50 \% geklettert und damit wieder in die Nähe des Wertes von 2003 (53 \%) gerückt. Die Zahl für Deutschland liegt dabei immer noch deutlich unter dem EU-Durchschnitt, der sich ebenfalls, wenn auch etwas weniger sprunghaft, erhöht hat (von 52 auf $56 \%$ ).

Männer sind in Deutschland praktisch genauso häufig wie Frauen (51 bzw. 50 \%) von Stress durch Spannungen zwischen Beruf und Privatleben betroffen, im Gegensatz zu den meisten anderen Ländern, wo Konflikte dieser Art von Frauen beträchtlich häufiger genannt werden als von Männern. Dies bedeutet eine Veränderung gegenüber 2007, als sich in Deutschland nur $41 \%$ der Männer und $47 \%$ der Frauen im Zusammenhang mit der Work-Life-Balance in mindestens einem der Bereiche Stress ausgesetzt sahen.

In Deutschland beteiligen sich die Männer stärker an den Haushaltsarbeiten als in den anderen Ländern Europas, übernehmen aber immer noch nicht den gleichen Anteil wie die Frauen: 65 \% der Männer und $92 \%$ der Frauen erledigen mehrmals pro Woche das Kochen oder andere Haushaltsarbeiten. Sowohl Männer als auch Frauen sind mit ihrem Anteil an der Hausarbeit deutlich zufriedener als der europäische Durchschnitt: Insgesamt $72 \%$ meinen, dass ihr Anteil ungefähr gerecht ist, verglichen mit nur $56 \%$ in der gesamten EU. Dieser Befund deutet darauf hin, dass Frauen in Deutschland zwar mehr Hausarbeit als Männer übernehmen, aber von dieser Ungleichheit weniger belastet sind als die übrigen Europäerinnen. Tatsächlich halten 75 \% der Frauen, die sehr viel Hausarbeit erledigen, dies für einen fairen Anteil (analog halten auch 76 \% der Männer mit hohem Hausarbeitspensum dies für fair). Diese positive Haltung zur häuslichen Arbeitsteilung bei Frauen (und Männern), die viel Hausarbeit machen, übertrifft den europäischen Durchschnitt von 58 \% bei Frauen (und 64 \% bei Männern).

Die berufliche Zufriedenheit ist gestiegen und wurde auf einer Skala von 1 bis 10 Punkten mit 7,6 bewertet (2007: 7,3). Gleichzeitig sank aber die Zufriedenheit mit dem Familienleben von 8,0 in 2007 auf 7,7 in 2011. Dies entspricht der allgemeinen Entwicklung in der Union, wo die Befragten 2011 mit ihrer Beschäftigung ebenfalls deutlich zufriedener waren als im Jahr 2007 (7,4 bzw. 7,1). Der Abwärtstrend bei der Zufriedenheit mit dem Familienleben hält andererseits schon länger an: 2003 lag die Bewertung noch bei 8,3 .

Sind die Befragten unter Berücksichtigung der Notwendigkeit, ihren Lebensunterhalt zu verdienen, mit der Anzahl 
ihrer Arbeitsstunden zufrieden? Vor dem Hintergrund bestimmter Entwicklungen im Bereich der Arbeitszeit kommt dieser Frage besondere Bedeutung zu: Als Reaktion auf die Wirtschaftskrise wurde 2009 in Deutschland den Unternehmen die Möglichkeit eingeräumt, durch Kürzung der Arbeitsstunden Arbeitsplätze zu erhalten. Dabei gewährt der Staat den betroffenen Arbeitnehmern Kurzarbeitergeld als Teilausgleich für die Arbeitszeitkürzung (Eurofound 2009).

Die EQLS zeigt, dass $12 \%$ der Beschäftigten ihre wöchentliche Arbeitszeit gerne erhöhen würden. ${ }^{4}$ Der Anteil fällt geringer aus als in der EU insgesamt, wo er $14 \%$ beträgt. Andererseits ist unter den Nichtbeschäftigten der Wunsch nach Arbeit stärker ausgeprägt als in allen anderen Ländern der EU: 77 \% der Betroffenen in Deutschland würden gerne zumindest einige Stunden arbeiten, in der gesamten EU sind es $72 \%$.

Frauen in Deutschland sagen häufiger als Männer und auch häufiger als Frauen in der übrigen EU, dass sie gerne mehr Stunden arbeiten würden; dagegen sind die Anteile jener Männer und Frauen gleich, die sich wünschen, weniger arbeiten zu können (Tabelle 1).

\section{Wohneigentum, Wohnbedingungen und Wohnumfeld}

Die Beschaffenheit des lokalen Wohnumfelds stellt einen wichtigen Aspekt der Lebensbedingungen dar. Die EQLS geht auf qualitative Aspekte des Wohnumfelds, der Unterkunft als solcher und der Wohnbedingungen im weiteren Sinne ein. Neben Fragen zu qualitativen Aspekten des lokalen Wohnumfelds wurde auch nach der Zugänglichkeit von Dienstleistungsangeboten im Wohngebiet gefragt.

\subsection{Wohneigentum und Wohnbedingungen}

In Deutschland ist es üblicher als in allen anderen Ländern der EU, zur Miete zu wohnen. Das gilt vor allem für den privaten Wohnungsmarkt, auf den $36 \%$ aller befragten Haushalte entfallen. Hinsichtlich der Qualität der Unterkünfte schneidet Deutschland bei den meisten Kriterien überdurchschnittlich ab. Die Befragten sind mit ihrem Wohnraum zufriedener, klagen weniger über Probleme mit Fäulnis (4 gegenüber $9 \%$ für die EU) und Feuchtigkeit (7 gegenüber $12 \%$ für die EU), haben nur in seltensten Fällen keine eigene Toilette oder kein eigenes Bad (jeweils $1 \%$ im Vergleich zu $3 \%$ in der EU) und berichten etwas seltener als anderswo von Platzmangel. Andererseits beklagt sich in Deutschland eine etwas höhere Anzahl (15\%) als im Durchschnitt der EU (14 \%) darüber, dass sie keinen Platz zum Sitzen im Freien haben. Mit ihrer Unterkunft zeigten sich die Befragten 2011 etwas weniger zufrieden (7,7) als im Jahr 2007 (7,9). In der übrigen EU ist die Zufriedenheit mit den eigenen Wohnverhältnissen dagegen leicht gestiegen: von 7,6 auf 7,7.
TABELLE 1

\section{Arbeitszeitwünsche Beschäftigter}

Angaben in Prozent

\begin{tabular}{|c|c|c|c|c|}
\hline & & $\begin{array}{l}\text { Würde gerne } \\
\text { mehr arbeiten }\end{array}$ & $\begin{array}{c}\text { Würde gerne } \\
\text { weniger arbeiten }\end{array}$ & $\begin{array}{l}\text { Würde bei der } \\
\text { derzeitigen Stunden- } \\
\text { zahl bleiben }\end{array}$ \\
\hline \multirow{2}{*}{ Deutschland } & Männer & 8 & 37 & 55 \\
\hline & Frauen & 17 & 37 & 46 \\
\hline \multirow{2}{*}{ EU27 } & Männer & 12 & 42 & 45 \\
\hline & Frauen & 15 & 43 & 42 \\
\hline
\end{tabular}

Quelle: Europäische Erhebung zur Lebensqualität (EQLS); Eurofound (2012a).

\subsection{Qualität des Wohnumfelds}

In Deutschland berichtet eine relativ geringe Zahl von Leuten von Problemen mit der Trinkwasserqualität (14 gegenüber $21 \%$ in der EU). Lärm wird dagegen öfter als Problem gesehen als in allen anderen Teilen der EU (42 gegenüber $33 \%$ in der EU). Hinsichtlich Problemen mit Abfall und Unrat auf Straßen und öffentlichen Plätzen (32\%), mit Kriminalität, Gewalt oder Vandalismus (34\%) sowie mit der Luftqualität (27 \%) liegen die Werte jeweils dicht am europäischen Durchschnitt.

\subsection{Zugang zu Dienstleistungen im Wohngebiet}

In Deutschland berichten die Befragten relativ häufig von Schwierigkeiten mit der Erreichbarkeit von Poststellen, und zwar nicht nur in ländlichen Raum (24 gegenüber $20 \%$ in der EU), sondern auch in den Städten (18 gegenüber $16 \%$ in der EU). Den Zugang zu Banken empfinden dagegen besonders auf dem Lande (17 gegenüber $22 \%$ in der EU), aber auch im städtischen Raum (11 gegenüber $13 \%$ in der EU) vergleichsweise wenige Leute als problematisch. Gründe für die verschiedene Wahrnehmung könnten Unterschiede in der Erwartungshaltung sein, aber ebenso die hohe Verfügbarkeit von Online-Diensten der Banken im Vergleich zu den beschränkten Möglichkeiten des Online-Zugriffs auf die Dienstleistungen der Post.

Den Zugang zu Naherholungsgebieten und Grünflächen empfinden Stadtbewohner in Deutschland vergleichsweise häufig als problematisch (16 gegenüber $13 \%$ in der EU), während im ländlichen Raum das Verhältnis umgekehrt ist (11 gegenüber $14 \%$ in der EU). Der Anteil der Einwohner deutscher Städte, die von Schwierigkeiten berichten,

4 In Fällen, in denen die Wunschvorstellung um zwei Stunden oder weniger pro Woche von der derzeitigen Arbeitszeit abwich (nach oben oder unten), wurde vermerkt, dass die befragte Person bei der derzeitigen Stundenzahl bleiben möchte. 
Kinos, Theater oder Kulturzentren zu besuchen, übersteigt mit $24 \%$ den Durchschnittswert der EU (20 \%). Im ländlichen Raum ist diese Problematik noch ausgeprägter: 59 \% der Landbewohner Deutschlands und 48 \% der Landbewohner der gesamten EU finden es schwierig, Angebote der genannten Art wahrzunehmen. Nur von den Bewohnern ländlicher Gebiete in Bulgarien, Griechenland, Polen, Portugal und Rumänien wird der Zugang zu diesen Angeboten noch häufiger als schwierig bezeichnet.

\section{6. Öffentliche Dienste, Gesundheits- wesen und Gesundheitszustand}

\section{1 Öffentliche Dienste: Zugänglichkeit und Qualität}

Die Befragten wurden aufgefordert, die Qualität verschiedener öffentlicher Dienste auf einer Skala von 1 (sehr schlecht) bis 10 (sehr gut) zu bewerten. In Deutschland zeigten sich die Befragten vor allem mit den öffentlichen Verkehrsmitteln zufrieden (7,0 im Vergleich zu 6,4 für die gesamte EU). Nur in Luxemburg und Österreich werden höhere Noten vergeben.

Im Zeitraum 2007 - 2011 hat sich zudem die Bewertung verschiedener öffentlicher Dienste verbessert: für das deutsche Bildungswesen von 5,8 auf 6,5, für die Kinderbetreuungseinrichtungen von 6,3 auf 6,6, für das staatliche Rentensystem von 4,5 auf 5,3 .

Hinsichtlich des Zugangs zu Kinderbetreuungseinrichtungen werden im Vergleich zur EU folgende Faktoren weniger häufig als problematisch empfunden: Kosten (50 gegenüber $59 \%$ in der EU), Qualität (25 gegenüber $27 \%$ in der EU), Erreichbarkeit beziehungsweise Öffnungszeiten (39 gegenüber $41 \%$ in der EU). Die Verfügbarkeit (z. B. Wartelisten, Fehlen von Betreuungsleistungen) wird als erschwerender Faktor öfter genannt (61 \%) als in der EU insgesamt (58\%).

Bei den Langzeitpflegediensten stellt sich das Bild etwas anders dar. Die Kosten dieser Dienste werden von einem höheren Anteil der Befragten (67 \%) als erschwerender Faktor gesehen als in der übrigen EU (60 \%). Betreuungsqualität (39 gegenüber $44 \%$ in der EU), Erreichbarkeit beziehungsweise Öffnungszeiten (41 gegenüber $49 \%$ in der EU) und Verfügbarkeit (57 gegenüber $61 \%$ in der EU) werden weniger häufig als in anderen Teilen der EU zu den Umständen gezählt, welche die Inanspruchnahme dieser Dienste erschweren.

\subsection{Gesundheitsversorgung}

Die Befragten in Deutschland benoten ihr Gesundheitssystem mit durchschnittlich 6,6 von maximal 10 Punkten deutlich besser als im Jahr 2007 (6,0). Der Anstieg entspricht der Qualitätsverbesserung, die auch bei anderen öffentlichen
Diensten wahrgenommenen wurde. Die Zugänglichkeit (Erreichbarkeit, Öffnungszeiten) scheint sich ebenfalls allgemein verbessert zu haben, wenngleich viele der Befragten noch immer von Schwierigkeiten bei der Inanspruchnahme von Gesundheitsdiensten berichten. Im Zusammenhang mit dem Besuch von Ärzten oder medizinischen Spezialisten nannten die Befragten in Deutschland weniger häufig die Behandlungskosten (25 gegenüber $30 \%$ in der EU) beziehungsweise die Entfernung zur Arztpraxis/zum Krankenhaus/vom medizinischen Zentrum (21 gegenüber $22 \%$ in der EU) als erschwerende Faktoren. Wartezeiten auf einen Termin (39 \%) und zeitliche Engpässe aufgrund beruflicher Verpflichtungen, wegen Kinderbetreuungsproblemen oder aus ähnlichen Gründen wurden ebenso häufig als Erschwernisse genannt wie in der gesamten EU (27 \%). Wartezeiten in der Praxis werden häufiger als in anderen Teilen der EU als problematisch bezeichnet: $48 \%$ der Befragten, die einen Arzt oder medizinischen Spezialisten aufgesucht hatten, sagten, die Wartezeit habe ihnen den Arztbesuch etwas oder sehr erschwert. In der gesamten EU sind es $42 \% .2007$ lag der Anteil in Deutschland noch bei $46 \%$.

\subsection{Gesundheitszustand}

2011 bezeichneten $9 \%$ der Befragten in Deutschland die eigene gesundheitliche Verfassung als schlecht oder sehr schlecht, gegenüber $8 \%$ im Jahr 2007. Der Anstieg betrifft vor allem Angehörige des untersten Einkommensviertels. Hier stieg der Anteil von 14 auf $17 \%$. Ein ähnlicher Trend lässt sich bei der Zufriedenheit mit der eigenen Gesundheit beobachten. In der untersten Einkommensgruppe sank die Zufriedenheit mit dem eigenen Gesundheitszustand von 7,1 auf 6,6 der maximal 10 Punkte; im zweitniedrigsten Einkommensviertel schrumpfte sie von 7,2 auf 7,0. Bei der besser verdienenden Bevölkerungshälfte dagegen entwickelte sich die Zufriedenheit mit der eigenen Gesundheit positiv. Der Trend lässt sich mit der Entwicklung in der gesamten EU27 vergleichen, ist in Deutschland aber deutlicher ausgeprägt (Tabelle 2).

Wie auch in anderen Ländern Europas gibt eine höhere Anzahl von Befragten an, unter chronischen körperlichen oder seelischen Gesundheitsproblemen, Krankheiten oder Behinderungen zu leiden. In Deutschland stieg der Anteil zwischen 2007 und 2011 von 28 auf $31 \%$, in der EU27 von 24 auf $28 \%$. Unter denjenigen, die Gesundheitsprobleme oder Behinderungen dieser Art angaben, fühlte sich 2011 ein höherer Anteil ( $83 \%)$ in seinen alltäglichen Aktivitäten beeinträchtigt als im Jahr 2007 (79\%).

\section{Zustand der Gesellschaft}

Die Betrachtung einer Indikatorenreihe, welche die zwischenmenschlichen Beziehungen und das Zugehörigkeits- 
TABELLE 2

\section{Zufriedenheit mit der eigenen Gesundheit}

Angaben auf einer Skala von 1 (unzufrieden) - 10 (zufrieden)

\begin{tabular}{|c|c|c|c|c|c|c|}
\hline \multirow[b]{2}{*}{ Einkommensgruppe } & \multicolumn{3}{|c|}{ Deutschland } & \multicolumn{3}{|c|}{ EU27 } \\
\hline & 2007 & 2011 & Veränderung & 2007 & 2011 & Veränderung \\
\hline unteres Quartil & 7,1 & 6,6 & $-0,5$ & 6,9 & 6,7 & $-0,3 *$ \\
\hline untere Mitte & 7,2 & 7,0 & $-0,2$ & 7,1 & 7,1 & $-0,1^{*}$ \\
\hline obere Mitte & 7,4 & 7,5 & 0,1 & 7,4 & 7,5 & 0,1 \\
\hline oberes Quartil & 7,7 & 7,9 & $0,3^{*}$ & 7,8 & 7,8 & 0,0 \\
\hline
\end{tabular}

*Veränderung ist auf Auf- bzw. Abrundung zurückzuführen.

Quelle: Europäische Erhebung zur Lebensqualität (EQLS); Eurofound (2012a).

gefühl zur Gesellschaft beschreibt, ergibt für Deutschland im Vergleich zur übrigen EU ein sehr positives Bild.

\subsection{Soziale Ausgrenzung}

Das Gefühl sozialer Ausgrenzung ist in Deutschland allgemein weniger stark verbreitet als in der EU insgesamt. Nur $9 \%$ der Deutschen fühlen sich „aus der Gesellschaft ausgeschlossen“, der EU-Durchschnitt beläuft sich auf $11 \%$. Für bestimmte Gruppen fällt der Unterschied bei diesem Indikator besonders groß aus: für Alleinerziehende (7 \% fühlten sich ausgegrenzt, gegenüber einem EU-Durchschnitt von $14 \%$ ) und für Menschen ab 70 Jahren (7 gegenüber $11 \%$ ). Das Gefühl sozialer Ausgrenzung wurde mit drei weiteren Indikatoren ${ }^{5} \mathrm{zu}$ einem Index zusammengefasst, der die soziale Ausgrenzung auf einer Skala von 1 bis 5 misst. Deutschland erzielt auf dieser Skala mit durchschnittlich 1,8 Punkten den zweitniedrigsten Wert in der gesamten EU (nach Dänemark) und liegt deutlich unter dem EU-Durchschnittswert von 2,2.

\subsection{Vertrauen in andere Menschen und in Einrichtungen}

Das Vertrauen in die Mitmenschen liegt, auf einer 10-Punkte-Skala gemessen, bei 5,0 und unterscheidet sich nicht wesentlich vom Durchschnittswert der EU. In den meisten Ländern der Erhebung war seit 2003 ein signifikanter Vertrauensschwund zu beobachten, so auch in Deutschland (-0,6 Punkte). Deutschland ist jedoch eines der Länder, in denen dieser Abwärtstrend zum Stehen kam und sich seit 2007 sogar wieder umgekehrt hat (um 0,2 Punkte).

Sowohl die Deutschen als auch andere Europäer legen gegenüber den politischen Institutionen und deren Vertretern eine eher kritische Haltung an den Tag; gleichzeitig nimmt die Wahlbeteiligung ab (Bertelsmann Stiftung 2012). Dennoch ist das Vertrauen in öffentliche Einrichtungen in Deutschland stärker ausgeprägt als im EU-Durchschnitt (Tabelle 3). Die Differenz gegenüber den durchschnittlichen EU-Werten ist für alle Gesellschaftsgruppen konstant.
Dieses größere Institutionenvertrauen steht möglicherweise in Zusammenhang mit dem höheren subjektiven Wohlbefinden (Watson et al. 2009) und dem niedrigeren Korruptionsempfinden (Eurofound 2012a). Beim Vertrauen in die Justiz und das nationale Parlament wird der Unterschied zum EU-Durchschnitt besonders deutlich - obwohl zum Zeitpunkt der Befragung die letzte Bundeswahl mehr als zwei Jahre zurücklag.

Das Vertrauensniveau variiert mit Einkommensgruppen. Das untere Einkommensquartil in Deutschland kommt beim Vertrauen in die Regierung auf einen Wert von 4,7, das obere auf 5,0. Enger als mit dem Einkommen hängt das Institutionenvertrauen mit der Fähigkeit zusammen, mit dem Einkommen auszukommen. Unter denjenigen, die angeben, nur mit Mühe über die Runden zu kommen, betrug das gemessene Vertrauen 3,7, unter denjenigen, die hier keine Schwierigkeiten berichteten, 5,3. Demnach vertrauen Menschen, die nur schwer mit ihrem Einkommen auskommen, in Deutschland ihrer Regierung im Mittel weniger als die entsprechende Gruppe in einigen anderen, stärker krisenbetroffenen Ländern wie Irland $(3,9)$ oder Estland (4,5).

\subsection{Soziale Spannungen}

Im Vergleich zu den meisten europäischen Ländern werden gesellschaftliche Spannungen in Deutschland als gering empfunden. Besonders groß ist der Unterschied bei der Frage, ob nach Wahrnehmung der Befragten Spannungen zwischen Menschen unterschiedlicher sexueller Orientierung bestehen: Nur $29 \%$ erkennen in diesem Zusam-

5 Wortlaut: „Das Leben ist heutzutage so kompliziert geworden, dass ich mich kaum noch zurechtfinde”, „Ich habe das Gefühl, dass die Leute in meiner Umgebung den Wert meinerTätigkeiten nicht anerkennen“ und „Einige Menschen sehen wegen meiner Arbeit oder meines Einkommens auf mich herab". 
TABELLE 3

\section{Vertrauen in öffentliche Einrichtungen}

Angaben auf einer Skala von 1 (niedrig) - 10 (hoch)

\begin{tabular}{|c|c|c|c|c|c|}
\hline & Parlament & Justiz & Polizei & Regierung & $\begin{array}{c}\text { Kommunal-/ } \\
\text { Stadtverwaltungen }\end{array}$ \\
\hline Deutschland & 5,2 & 6,0 & 6,8 & 4,9 & 5,9 \\
\hline EU-Durchschnitt & 4,1 & 4,8 & 6,0 & 4,0 & 5,2 \\
\hline
\end{tabular}

Quelle: Europäische Erhebung zur Lebensqualität (EQLS); Eurofound (2012a).

menhang "große Spannungen“, im Gegensatz zu einem EUDurchschnitt von $43 \%$. Ebenso sehen nur $29 \%$ der Befragten (gegenüber $37 \%$ in der EU) Spannungen zwischen Volks- oder ethnischen Gruppen.

Die Spannungen zwischen Arm und Reich werden ebenfalls als weniger gravierend empfunden als im Durchschnitt der EU: 32 \% der Deutschen und durchschnittlich $35 \%$ aller EU-Bürger betrachten diese Spannungen als groß. In der EU insgesamt stieg der entsprechende Anteil im Zeitraum 2007 - 2011 von 30 auf $41 \%$, in Deutschland sank er von 36 auf $32 \%$.

Allerdings gibt es zwei Mitgliedstaaten, in denen die Spannungen zwischen Arm und Reich von überdurchschnittlich vielen Menschen als groß empfunden werden: Ungarn (71 \%) und Litauen (60 \%). Diese Werte treiben den EU-Durchschnitt in die Höhe - tatsächlich werden die sozialen Spannungen in mehreren Mitgliedstaaten weniger stark empfunden als in Deutschland. Wie auch in anderen EU-Ländern empfinden besonders die untersten Einkommensempfänger die Spannungen zwischen Arm und Reich als groß (39\% des untersten Einkommensviertels gegenüber $28-30 \%$ der höheren Gruppen).

\section{Fazit}

Die Lebensqualität in Deutschland ist im Vergleich zu anderen EU-Mitgliedstaaten hoch. Dieser Befund gilt allerdings nicht für alle Dimensionen der Lebensqualität. Zudem gibt es auch innerhalb Deutschlands erhebliche Unterschiede. Es kann wohl nur ein kleinerer Teil der real gemessenen niedrigen Werte bei subjektiven Indikatoren mit vergleichsweise hohen Erwartungen erklärt werden. Mit Sicherheit bietet sich in diesen Feldern regelmäßig die Möglichkeit, Verbesserungen der Lebensqualität zu erreichen.

Wie angesichts des relativ hohen Wohlstands sowie des Wachstums der deutschen Wirtschaft in der letzten Zeit nicht anders zu erwarten, schneidet Deutschland in Bezug auf den Lebensstandard und die finanzielle Situation der Befragten besser ab als die übrige EU. Überraschenderwei- se hat sich jedoch die Quote materieller Entbehrung in den letzten Jahren kaum verbessert. Der Anteil der Haushalte, die angaben, finanziell nicht oder nur mit Mühe über die Runden zu kommen, hat sich erhöht. Eine mögliche Erklärung für die diesbezüglichen Umfrageergebnisse und den scheinbaren Widerspruch zur positiven Wirtschaftsentwicklung in Deutschland wäre, dass nur ein Teil der Bevölkerung vom Wirtschaftswachstum profitiert hat.

Auch Schulden scheinen in Deutschland ein größeres Problem zu sein, als man vielleicht erwarten würde. Unter den Ursachen der Verschuldung spielt Arbeitslosigkeit eine große Rolle. Zwar gibt es Anzeichen für eine Verbesserung der Lage, gleichzeitig war jedoch ein deutlicher Anstieg der gesundheitsbedingten Fälle von Überschuldung zu verzeichnen (Knobloch/Reiffner 2012). Der hohe Verbreitungsgrad informeller Zahlungsrückstände ist besonders besorgniserregend, da solche Rückstände unter Umstände die Erkennung von Überschuldungsproblemen hinauszögern. Sie können zudem soziale Beziehungen zerrütten (die angesichts finanzieller Schwierigkeiten für die Lebensqualität besonders wichtig sind) und können einen Domino-Effekt weiterer Zahlungsschwierigkeiten auslösen (Eurofound 2012c). ${ }^{6}$

Das subjektive Wohlbefinden hat sich über die letzten vier Jahre positiv entwickelt. Dabei hebt sich Deutschland von anderen EU-Ländern durch eine geringere Varianz des subjektiven Wohlbefindens zwischen den einzelnen Altersgruppen ab. Die Bevölkerung Deutschlands sieht besonders optimistisch in die Zukunft, und ihr Optimismus hat sich seit 2007 weiter verbessert.

Während die Zufriedenheit mit dem eigenen Familienleben langfristig abgenommen hat, ist die Zufriedenheit am Arbeitsplatz gestiegen. Diese Beobachtung gilt für viele Länder, in denen das Beschäftigungsangebot im Gefolge der Wirtschaftskrise knapper geworden ist. Befragte, die keiner bezahlten Beschäftigung nachgehen, zeigen sich in Deutschland häufiger an Arbeit interessiert als in der übrigen EU. Ebenso scheint sich Arbeitslosigkeit in Deutschland besonders negativ auf das subjektive Wohlbefinden auszuwirken.

Mit ihrer Work-Life-Balance und ihrem Anteil an der Hausarbeit zeigten sich die Befragten in Deutschland relativ zufrieden; in beiden Aspekten ist die Verteilung zwischen den Geschlechtern ausgewogener als im EU-Durchschnitt. Geschlechtergerechtigkeit ist stärker ausgeprägt als in der EU und sie hat sich in einigen Dimensionen seit 2007 verbessert. Trotzdem erledigen Frauen deutlich mehr Hausarbeit als Männer, halten diese Arbeitsteilung aber im Gegensatz zu Frauen in anderen EU-Ländern eher für fair.

In Deutschland wird die Qualität öffentlicher Dienste bedeutend besser beurteilt als in den meisten EU-Ländern; die Einschätzung ist in den letzten vier Jahren sogar noch

6 In dieser jüngeren Untersuchung befasste sich Eurofound mit der Frage, welche Maßnahmen den Betroffenen und Interessenten zur Verfügung stehen, um dem Problem der Überschuldung von Haushalten zu begegnen. 
positiver geworden. Der Zugang zu Kinderbetreuungseinrichtungen, Langzeitpflegediensten und dem Gesundheitssystem wird allgemein als leichter empfunden als im Durchschnitt der EU. Andererseits geben im Zusammenhang mit der Inanspruchnahme dieser Dienste die Langzeitpflegekosten und die Wartezeiten bei Ärzten potenziell Anlass zu Besorgnis.

Insgesamt empfindet die Bevölkerung Deutschlands den eigenen Gesundheitszustand als gut; hier hat es relativ geringe Veränderungen gegeben. Das Bild ist aber weniger günstig, wenn man nur die unterste Einkommensgruppe betrachtet. Im unteren Einkommensviertel bezeichnet ein relativ hoher Anteil der Befragten die eigene Gesundheitsverfassung als schlecht, und dieser Anteil ist stärker gestiegen als in anderen Ländern Europas.

Bei den Indikatoren für gesellschaftliche Lebensqualität rangiert Deutschland über dem EU-Durchschnitt. Dies drückt sich besonders in einem höheren Vertrauen in die öffentlichen Institutionen und in der geringeren Wahrnehmung von sozialen Spannungen aus. Dennoch sind die Spannungen zwischen Arm und Reich vergleichsweise stark - ein Umstand, der besonders im Zusammenhang mit den beobachteten Ungleichheiten in den Bereichen Gesundheit, Lebensstandard und finanzielle Situation für die Politik von Interesse sein dürfte. ${ }^{7}$

7 Hinsichtlich des Zusammenhangs zwischen sozialer Ungleichheit und Lebensqualität wird Eurofound Mitte 2013 eine vertiefende Analyse der EQLS-Daten veröffentlichen.

\section{LITERATUR}

Bertelsmann Stiftung (2012): Cohesion Radar: Measuring Cohesiveness Social Cohesion in Germany - a preliminary Review, Gütersloh

European Foundation for the Improvement of Living and Working Conditions (Eurofound) (2009): New allowances for short-time work in bid to offset economic crisis, http://www.eurofound.europa.eu/eiro/2009/04/articles/ de0904039i.htm
European Foundation for the Improvement of Living and Working Conditions (Eurofound) (2012a): Third European Quality of Life Survey - Quality of life in Europe: Impacts of the crisis: Publications Office of the European Union, Luxembourg, http://www.eurofound.europa.eu/publications/htmlfiles/ef1264.htm European Foundation for the Improvement of Living and Working Conditions (Eurofound) (2012b): Income from work after retirement in the EU, http://www. eurofound.europa.eu/publications/htmlfiles/ef1259.htm

European Foundation for the Improvement of Living and Working Conditions (Eurofound) (2012c): Household debt advisory services in the European Union http://www.eurofound.europa.eu/publications/htmlfiles/ef1189.htm Eurostat (2013): Online database, http://epp.eurostat.ec.europa.eu/tgm/table. do tab=table\&init=1\&plugin $=1 \&$ language $=e n \&$ pcode $=$ tec00115, accessed 7 February

Knobloch, M./Reiffner, U. (2012): Überschuldung in Deutschland: Institut für Finanzdienstleistungen e. V., iff-Überschuldungsreport, Hamburg Watson, D./Pichler, F./Wallace, C.: (2009):, Subjective well-being in Europe: Eurofound, http://www.eurofound.europa.eu/pubdocs/2009/108/en/1/ EF09108EN.pdf

\section{AUTOREN}

HANS DUBOIS ist Research Officer bei der Europäischen Stiftung zur Verbesserung der Lebens- und Arbeitsbedingungen (Eurofound) in Dublin. Arbeitsschwerpunkte: Lebensbedingungen und Lebensqualität.

hdu.dubois@eurofound.europa.eu

ESZTER SANDOR ist Research Officer bei Eurofound. Arbeitsschwerpunkte: Lebensbedingungen und Lebensqualität.

eszter.sandor@eurofound.europa.eu

ERIKA MEZGER, Dr. rer. soc, ist Deputy Direktor (Vizedirektorin) von Eurofound.

erika.mezger@eurofound.europa.eu 